\title{
Pathogen interactions with cytokines and host defence: an overview
}

\begin{abstract}
This review summarises some of the immune evasion tactics adopted by pathogens. They include the antagonism of immune function through the use of homologues of cytokine receptors, expression of viral proteins which interact with cytokine signal transduction and expression of cytokine mimics and host proteins that influence the Type I or II cytokine responses. Some of the viral defense molecules that interfere with the functions of cytokines include the EBV protein BCRF1 (viral IL-10) which blocks synthesis of cytokines such as IFN-כ, viral IL-17 and IL-8 receptor encoded by the herpesvirus saimiri genome and chemokine receptor homologues of Epsteinï Barr virus, herpesvirus saimiri and cytomegalovirus. These immunomodulatory tactics function to protect the host from the lethal inflammatory effects as well as inhibit the local inflammatory response elicited to kill the foreign pathogen. Other strategies include the alterations in cytokine expression such as demonstrated with the hepatitis B virus (HBV) core protein and terminal protein which can inhibit interferon- $\delta$ gene expression, the interactions of the hepatitis $C$ virus core protein to lymphotoxin- 6 receptor and the effects of the interferon signal transduction pathway by adenovirus EIA oncogene and HBV by reducing levels or activity of the cytosolic latent transcriptional factors (STATS). Immune evasive strategies of helminth parasites related to cytokine activities will also be briefly discussed.
\end{abstract}

Keyword: Pathogen; Cytokines; Viral proteins; Herpesvirus saimiri; Epstein Barr virus; Cytomegalovirus 